\begin{tabular}{l|l} 
REVISTA Revista Educación \\
EDUEACIÓN
\end{tabular}$\quad \begin{aligned} & \text { ISSN: 0379-7082 } \\
& \text { ISSN: 2215-2644 } \\
& \text { revedu@gmail.com } \\
& \text { Universidad de Costa Rica } \\
& \text { Costa Rica }\end{aligned}$

\title{
Cuantificación de la Inequidad Educativa en América Latina (2000-2015)
}

\author{
Formichella, María Marta \\ Cuantificación de la Inequidad Educativa en América Latina (2000-2015) \\ Revista Educación, vol. 44, núm. 2, 2020 \\ Universidad de Costa Rica, Costa Rica \\ Disponible en: http://www.redalyc.org/articulo.oa?id=44062184009 \\ DOI: https://doi.org/10.15517/revedu.v44i2.38413
}

Esta obra está bajo una Licencia Creative Commons Atribución-NoComercial-SinDerivar 3.0 Internacional 


\title{
Cuantificación de la Inequidad Educativa en América Latina (2000-2015)
}

\author{
Educational Inequality Quantification in Latin America (2000-2015) \\ María Marta Formichella \\ Universidad Nacional del Sur, Argentina \\ DOI: https://doi.org/10.15517/revedu.v44i2.38413 \\ Redalyc: http://www.redalyc.org/articulo.oa?id=44062184009
}

mformichella@iiess-conicet.gob.ar

(iD) http://orcid.org/0000-0002-2057-0938
Recepción: 02 Septiembre 2019

Aprobación: 20 Febrero 2020

\section{Resumen:}

El objetivo del trabajo es cuantificar la inequidad educativa en América Latina en los años 2000, 2003, 2006, 2009,2012 y 2015 , determinar qué países se encuentran mejor situados en cada período y si existen cambios en dichos posicionamientos. Para ello, se utiliza un índice sintético ponderado el Índice de Inequidad Educativa Básica propuesto por Formichella (2014)-, las Encuestas de Hogares procesadas por el Centro de Estudios Distributivos, Laborales y Sociales (CEDLAS) y el Programa Internacional de Evaluación de Alumnos (PISA). El índice se calcula para los siguientes países: Argentina, México, Perú, Brasil, Chile, Uruguay, Colombia, Panamá, Costa Rica y República Dominicana. Se concluye que, si bien la inequidad educativa ha disminuido en la mayoría de los países estudiados, el ranking de ordenamiento no se ha modificado y los niveles de inequidad aún son elevados. Se recomienda continuar con la recolección de información y el cálculo de la inequidad educativa, por ser útil al momento de ejecutar políticas.

Palabras Clave: Inequidad Educativa, América Latina, Educación Secundaria, Índice de Equidad Educativa.

\section{Abstract:}

The objective of this study is to quantify educational inequality in Latin America in 2000, 2003, 2006, 2009, 2012 and 2015 , identify which countries had a better ranking during each period and where each country stands today with regards to that position. The assessment is based on a weighted synthetic index that includes data from the Basic Educational Inequity Index (IIEB-2014) proposed by Formichella (2014), a Household Survey conducted by the Center for Distributive, Labor and Social Studies (CEDLAS) and the Programme for International Student Assessment (PISA). The index was calculated for the following countries: Argentina, Mexico, Peru, Brazil, Chile, Uruguay, Colombia, Panama, Costa Rica and the Dominican Republic. It was concluded that despite a general reduction in educational inequality in most of the countries studied, the current ranking remains unchanged while the level of educational inequality still continues to remain high.

KEYWORDS: Educational Inequality, Latin America, Secondary Education, Educational Equity Index.

\section{INTRODUCCIÓN}

La educación es una capacidad esencial. Las personas con educación pueden optar con mayor libertad acerca del estilo de vida que desean llevar adelante (Sen, 1999, 2003) y las sociedades cuyos habitantes se encuentran educados pueden desarrollarse más favorablemente (Formichella, 2010, 2011). Es así que, los gobiernos latinoamericanos históricamente se han propuesto metas en materia de educación. En las décadas de los cincuenta y sesenta el objetivo estaba en combatir el analfabetismo y en la universalización de la educación primaria (UNESCO, 1966) y posteriormente estas se fueron ampliando. Así, incluyeron la universalización de la educación primaria y secundaria; el aumento de la participación de los jóvenes en la educación secundaria superior, técnico profesional y universitaria; la necesidad de mejorar la equidad y la calidad educativa; y la importancia de fortalecer la profesión docente, entre otras cuestiones (Torres, 2010).

Este trabajo centra la atención en la equidad educativa en la región latinoamericana. Su propósito es cuantificar la inequidad educativa en los años 2000, 2003, 2006, 2009, 2012 y 2015; asimismo, se intenta determinar un ranking en relación con qué países son los más equitativos en educación y si existen cambios 
en los posicionamientos en los diferentes años considerados. En síntesis, se busca describir el escenario de la equidad educativa en América Latina.

Específicamente, se considera a la equidad educativa desde el punto de vista interno y en el nivel educativo básico. Que se estudie la equidad interna significa que se pone la atención dentro del sistema educativo, a diferencia de la externa que se vincula a los logros posteriores al paso por este, relacionados principalmente con el mundo laboral (Calero, 1999). Por otra parte, la educación básica hace referencia a la educación de nivel medio.

Asimismo, cabe señalar que siguiendo a Formichella $(2011,2014)$, se analiza la equidad educativa interna desde la perspectiva de los logros escolares. Es decir que -entre las diferentes bases de información que pueden elegirse para definir a la equidad educativa (acceso, medios de aprendizaje, logros, etc.)- se escoge hacerlo a partir de la igualdad en los resultados educativos que obtienen las personas. La elección de este enfoque se vincula al siguiente argumento: dado que la educación es esencial para el desarrollo de los individuos, es necesario que todos alcancen ciertos resultados mínimos para poder vivir su vida adulta en plenitud, lo cual implica que puedan desenvolverse favorablemente como trabajadores y ciudadanos (Sen, 1999).

Definir a la equidad educativa desde la perspectiva de los resultados es totalmente compatible con el enfoque del acceso y las oportunidades; asimismo, es más amplio y significa que todos los individuos acceden al sistema y, una vez allí, sus diferencias de origen son compensadas para que todos puedan alcanzar los logros mínimos establecidos como deseables (Morduchowicz, 2003). Asimismo, esta mirada es superadora a los enfoques de la igualdad en los insumos o en los medios de aprendizaje, perspectivas que consideran que existe equidad si todos reciben los mismos recursos, dejando de lado que no todos tienen las mismas capacidades para convertir dichos recursos en logros e instaurando una lógica meritocrática que culpabiliza al estudiantado que no puede alcanzar los resultados esperados (López, 2006).

Con respecto a la metodología, para cumplir con el objetivo del trabajo se utiliza un indicador sintético: el Índice de Inequidad Educativa Básica (IIEB) propuesto por Formichella (2014). Sin embargo, se introducen cambios en el mismo con el fin de mejorar la cuantificación de la equidad. De este modo, el trabajo se presenta como una investigación de tipo descriptiva del fenómeno en cuestión (de forma análoga a las investigaciones que estudian la desigualdad en el ingreso económico).

Finalmente, el trabajo se estructura así: en la sección dos se explicita el marco teórico-conceptual, en la siguiente se exponen los antecedentes sobre el tema; en el apartado cuatro se presentan los datos y las variables utilizados y se explicita la metodología; en el cinco se exhiben los resultados obtenidos y, posteriormente, en el seis se da paso a las conclusiones del trabajo.

\section{MARCo TEÓRICO-CONCEPTUAL}

En la literatura se encuentran diferentes conceptualizaciones de la equidad educativa. Comprenderlas, en especial entender cuáles son sus implicancias, es sumamente relevante (López, 2006).

Las diversas formas de definir la equidad educativa provienen del significado de la palabra equidad. Este vocablo deriva del latín -aequitas- y quiere decir igual; y por ello, a partir de cuál sea el elemento elegido como igualador entre las personas, se han desarrollado distintas teorías de la justicia social (Formichella, 2011).

Algunos autores planten propuestas basadas en que la equidad se verifica si existe igualdad en el bienestar cuantificado a través de la idea de utilidad (welfaristas) y otros, los denominados post-welfaristas, critican la utilización de esta unidad de medida y proponen el uso de diferentes opciones (Fleurbaey, 1995). De este modo, al interior de este segundo grupo se encuentran las ideas fundadas en la igualdad de los bienes, con autores como Rawls $(1971,1999)$ y Dworkin (1981), y las que se centran en la igualdad de las oportunidades, representadas por Sen (1979, 1999), Cohen (1989), Roemer (1995) y Arneson (citado en Ribotta, 2006). 
Cabe destacar que, Sen (1979) fue pionero en explicar claramente que la palabra equidad hace alusión a la igualdad, pero que el punto álgido a la hora de definir equidad es qué aspecto tener en cuenta como elemento de igualación.

En educación, Marc Demeuse (citado por López, 2006) postula cuatro posibles criterios de equidad: igualdad en el acceso; igualdad en los medios de aprendizaje; igualdad en los resultados; e igualdad en la organización social de estos. El primero, implica que si todos tienen oportunidad de asistir a la escuela existe equidad, sin embargo, no tiene en cuenta las diferencias que puedan suscitarse en el desenvolvimiento del estudiantado debido a sus discrepancias socioculturales. El segundo criterio, pone el foco en el modo de enseñanza y deja afuera, por un lado, la cuestión del acceso y, por otra parte, que no todos poseen las mismas cualidades para participar exitosamente de las clases. El tercer criterio, reconoce las diferencias iniciales y hace énfasis en que todos tengan el mismo acceso al conocimiento. Por último, el cuarto criterio establece que un sistema educativo es equitativo si en todos los escenarios sociales el impacto social de la educación es el mismo.

En este trabajo se sigue a Formichella (2011), quien analiza la equidad educativa a la luz del concepto de capacidades de Sen y considera que existe equidad educativa si hay igualdad en los resultados. En términos resumidos, se prefiere este enfoque en vez de aquellos que definen la equidad educativa a partir de la igualdad en el acceso, insumos o medios de aprendizaje porque estos últimos no tienen en cuenta que las personas difieren en lo que son capaces de hacer dentro del sistema educativo. Estos criterios consideran equitativo el igual trato a los iguales, es decir que implícitamente están suponiendo que el alumnado llega en condiciones homogéneas a la escuela. Sin embargo, asistir a la escuela, aún si cada estudiante accediera a los mismos insumos y medios educativos, no implica poder desenvolverse con éxito en ella (Morduchowicz, 2003; López, 2006).

Con respecto a los enfoques que definen equidad como igualdad en las oportunidades o como igualdad en los resultados, Morduchowicz (2003) explica que buscar la igualdad de resultados en educación no contradice a la idea analizar la igualdad de oportunidades, sino que la complementa, porque para obtener igualdad en resultados es necesario que se establezcan las condiciones necesarias para ello.

La principal diferencia entre estos enfoques se basa en la idea de la responsabilidad. Por una parte, la igualdad en las oportunidades considera que existen cuestiones vinculadas a las circunstancias que atraviesan las personas y cuestiones que no dependen de estas. Así, se hace explícito un límite a la igualación de resultados vinculado a la responsabilidad de los individuos en relación a los elementos que están bajo su control y solo debería existir compensación de aquellas circunstancias que escapan a la esfera dominada por las personas (Roemer, 1995). Por otra parte, la idea de la igualdad en los resultados cuestiona el grado de responsabilidad individual que pueda tener un individuo en edad escolar al efectuar sus elecciones; dado que en la niñez y adolescencia las decisiones de las personas están fuertemente influenciadas por su medio ambiente y circunstancias, quedando dudas acerca de cuán real es la posibilidad de elegir en esa etapa. Las personas adultas, especialmente su familia, son su guía y los contextos mediatos e inmediatos que los rodean influyen de manera determinante sobre sus condiciones de elección (Montes, 2004; Bolívar, 2005; Formichella, 2011). Así, podría decirse que su nivel de esfuerzo y responsabilidad está determinado por sus circunstancias y, por ello, aquí se considera más adecuado definir equidad educativa básica (que incluye nivel primario y medio, es decir alumnos entre 6 y 17 años) como igualdad en los resultados.

La dificultad de adoptar el enfoque de equidad educativa básica desde la perspectiva de los logros es la definición de los resultados deseables y cómo alcanzarlos (Morduchowicz, 2003). Este último punto se disipa como dificultad en este trabajo, cuyo objetivo se limita a cuantificar la equidad educativa básica y, entonces, se hace hincapié en cuáles son los resultados a considerar y cómo medirlos.

Siguiendo a Formichella (2014) se parte de la idea de que la igualdad absoluta no es viable y se busca un resultado común mínimo. Así, la igualdad en los logros implica que todo el alumnado pueda alcanzar ciertas aptitudes y actitudes para elegir qué estilo de vida desean tener (Formichella, 2011). 
Asimismo, también siguiendo a Formichella (2014), se define y cuantifica equidad interna en función de la educación secundaria de los individuos debido a que es la educación de nivel medio la considerada necesaria para que una persona se desarrolle en forma adulta en la esfera personal y ciudadana. Es decir que, el análisis se circunscribe a la equidad educativa básica y no se incorpora información acerca de los niveles superiores de educación.

En síntesis, en este trabajo se considera que hay equidad educativa interna básica si todos los individuos culminan el nivel medio (cantidad educativa: doce años de estudio aprobados) con ciertas competencias mínimas adquiridas (calidad educativa). La incorporación de la calidad es especialmente necesaria al estudiar América Latina, debido a que los títulos secundarios no son homogéneos (Formichella, 2014; Krüger, 2013).

\section{ANTECEDENTES}

Dado que en este trabajo se hace uso de un indicador de tipo sintético, aquí se expone una recopilación (no exhaustiva) de aquellas investigaciones en las cuales se ha presentado un índice de esta clase para medir la equidad educativa en países latinoamericanos. Un indicador sintético se define como aquel que combina información proveniente de indicadores individuales (Cabrer, Castro, y Pavía, 2001 citado por Mondéjar y Vargas, 2008), los cuales cuantifican un aspecto parcial del fenómeno que se pretende analizar (Mondéjar y Vargas, 2008).

Así, los indicadores sintéticos resultan útiles para salvar la dificultad de interpretar datos desagregados (Pérez et al., 2008), permiten describir el fenómeno bajo estudio de manera más sencilla y amplia (Mondéjar y Vargas, 2008) y hacen posible resumir información en un valor único, lo cual facilita la toma de decisiones (Pérez et al, 2008). En este mismo orden, Shavelson, McDonnell y Oakes (1991) resaltan que un indicador simple difícilmente podría dar información útil de un fenómeno complejo como es la educación.

Castillo (2003) presenta un Índice de Equidad Educativa (IEE) compuesto y lo aplica al caso de Honduras. El IEE relaciona la tasa de cobertura, la capacidad del sistema, la calidad del profesorado y la paridad de sexos. Para cuantificar estos conceptos, el autor utiliza cuatro indicadores educativos vinculados a insumos dentro de la función de producción educativa: a) tasa bruta de matrícula combinada b) relación estudiante-docente; c) porcentaje de docentes con título académico acorde al nivel en que se encuentra; y d) el índice de paridad de sexos (número de alumnas mujeres en relación al de varones dentro del sistema).

Luego, el índice compuesto se forma como promedio simple de los indicadores individuales estandarizados. Asimismo, se estandariza su valor con el fin de que el IEE tenga valor promedio cero y desvío estándar uno (IEE <-1: equidad baja; -1 < IIE< 1: equidad media; IEE > 1: equidad alta).

Gamboa y Waltemberg (2012) toman el enfoque de equidad de Roemer (igualdad en las oportunidades) y proponen un índice para estudiar la equidad educativa en un grupo de seis países latinoamericanos (Argentina, Brasil, Colombia, Chile, México y Uruguay). Tienen en cuenta que existen dos tipos de desigualdades en los resultados educativos: justa e injusta. La justa se debe a diferencias en el esfuerzo y la injusta a circunstancias que exceden el dominio de los individuos. Por ello, el índice que proponen indica cuál es el porcentaje de desigualdad injusta en relación a la desigualdad total. Las variables que consideran para cuantificar los resultados educativos tienen que ver con la calidad educativa, pues hacen uso de los resultados del Programa Internacional de Evaluación de Alumnos -PISA-, en las tres competencias evaluadas (matemática, lectura y ciencias).

Para estimar qué desigualdad es injusta y cuál es justa dividen al alumnado en grupos, donde en cada grupo hay un tipo de estudiante. Luego, al interior de cada uno se los divide de acuerdo con sus resultados. Así, se supone que las diferencias de logros entre quienes pertenecen al mismo grupo se deben a discrepancias en el esfuerzo. Mientras que las diferencias entre individuos pertenecientes a distintos grupos, pero que se hallan ubicados en el mismo segmento de la distribución de resultados dentro de cada grupo, se considera que son 
injustas. Al indicador que utilizan lo denominan Índice de Inequidad de las Oportunidades (IO) y lo estiman como la razón entre la Desigualdad al interior de los segmentos y la Desigualdad Total.

Por su parte, Chávez, Abreu y Charles (2013) proponen el Índice de Equidad Educativa Indígena (IEEI) para estudiar la cuestión en México. Para ello, toman elementos técnicos del Índice de Brecha de Género del Foro Económico Mundial, lo cual implica que la base el IEEI es la comparación de dos grupos poblacionales (en este caso indígenas y no-indígenas) por medio de razones. Es decir que, este índice no es útil a la hora de armar un ranking, ya que solo puede comparar una población determinada con otra.

Estos autores, con el fin de calcular un índice que tome en cuenta diferentes dimensiones de la inequidad que existe entre indígenas y no-indígenas, consideran cuatro componentes y diversos indicadores al interior de cada uno de ellos. Los componentes son: a) asistencia; b) escolaridad; c) Alfabetismo; y d) competencias consideradas por el programa PISA. Luego, calculan la brecha entre indígenas y no-indígenas correspondiente a cada indicador, estandarizan los valores y agregan los indicadores de cada componente como promedio no ponderado. Finamente, agregan los componentes para dar lugar al índice. En este caso primero estandarizan los valores de cada dimensión y luego elaboran un promedio simple de los resultados.

Formichella (2014) propone el Índice de Inequidad Educativa Básica (IIEB) para cuantificarla en Argentina, Brasil, Chile, México, Perú, Uruguay, Panamá y Colombia. Parte de considerar que existe equidad educativa cuando se verifica igualdad en los resultados educativos desde el punto de vista interno y se concentra en la equidad de la educación básica, hoy vinculada al nivel medio (Formichella, 2011). Además, toma en cuenta dos dimensiones referidas a los resultados: cantidad y calidad educativa. Dentro de la esfera de la cantidad educativa incluye dos indicadores: a) porcentaje de la población entre 25 y 65 años que no posee secundario completo y b) la tasa de extraedad en el nivel medio. Asimismo, como indicador de la calidad educativa también considera resultados del Programa PISA. Luego, el valor del IIEB surge como promedio ponderado de los tres indicadores mencionados: se propone un Índice de Ponderación del Analista (IPA) para que cada investigador explicite el peso relativo que le otorga a cada dimensión y a cada indicador.

Luego, Carvalho, Gamboa y Waltenberg (2015) y Gamboa y Waltenberg (2015) presentan un índice bidimensional, en el cual miden la equidad para los mismos seis países latinoamericanos que Gamboa y Waltemberg (2012), teniendo en cuenta no solo los logros educativos, sino también el acceso. Al igual que en el índice mencionado anteriormente, estiman cuál es el porcentaje de desigualdad injusta en los resultados educativos en relación a la desigualdad total $(\mathrm{IO})$ y, al mismo tiempo, incorporan una medición vinculada al acceso.

Por otra parte, si bien no fue aplicado en Latinoamérica, vale la pena mencionar que Assiego y Ubrich (2015) presentan un índice de equidad educativa al cual denominan Save the Children debido a que siguen la metodología que la organización Save de Children utiliza para calcular el denominado Índice de las Madres. Esta metodología consiste en elegir un conjunto de indicadores vinculados al fenómeno que se desea medir y luego hacer un ranking entre un grupo de países (u otra división geográfica) con cada uno de dichos indicadores. Luego, el índice compuesto se obtiene de hacer un promedio simple o ponderado con las posiciones que cada país obtuvo. Esta metodología tiene la ventaja de ser sencilla de llevar a cabo, pero carga con la desventaja de que el valor del índice es relativo y no absoluto. Es decir, un país con valor del índice igual a uno (el mejor posicionado en todos los indicadores) puede no tener un valor deseable del fenómeno que se está midiendo, solo implica que es el mejor del grupo bajo estudio.

El Índice de Equidad Educativa propuesto por Assiego y Ubrich (2015) considera catorce elementos vinculados al acceso a la educación en el ámbito escolar, y a la participación de las y los niños en actividades culturales y de ocio fuera del colegio. Estos autores calcularon el valor del Índice de Equidad Educativa para las 19 Comunidades o Ciudades Autónomas de España y presentaron el valor del índice para cada Comunidad o Ciudad Autónoma como un promedio simple de las posiciones obtenidas. 
Asimismo, Castro (2019) construye un índice de equidad educativa a partir de indicadores de inputs y outputs educativos, mediante la utilización de la técnica de análisis envolvente de datos. Luego, lo aplica para describir las diferencias entre las entidades territoriales en Colombia.

Como ya se ha mencionado, detrás de cada índice de equidad educativa existe una concepción de esta vinculada a cuál es el aspecto educativo que se considera debe ser igualitario para enunciar que se evidencia equidad.

Respecto a esto, se vislumbra que Castillo (2003) y Assiego y Ubrich (2015) se concentran en indicadores vinculados a la igualdad en el acceso, los insumos y los medios de aprendizaje. Chávez, et al. (2013) y Castro (2019) combinan insumos educativos con resultados educativos. Gamboa y Waltenberg (2012, 2015) y Carvalho, Gamboa y Waltenberg (2015) consideran que existe equidad si hay igualdad de oportunidades en educación. Mientras que Formichella (2014) hace referencia a la igualdad en los logros escolares.

\section{DAtos y Metodología}

\subsection{Datos}

Para cumplir con el objetivo del trabajo se utilizan las siguientes fuentes de información secundaria: datos provenientes de las Encuestas de Hogares de cada país, procesados por el Centro de Estudios Distributivos, Laborales y Sociales (CEDLAS) de la Universidad Nacional de la Plata, y datos del programa PISA, llevado a cabo por la Organización para la Cooperación y el Desarrollo Económicos (OCDE). Por ello, se cuantifica la equidad en los países latinoamericanos participantes de PISA: Argentina, México, Perú, Brasil, Chile, Uruguay, Colombia, Panamá, Costa Rica y República Dominicana.

Las encuestas de hogares son una fuente esencial de datos sociales y económicos. Tienen la ventaja de ser flexibles y de permitir que prácticamente todo tema vinculado a la población puede ser abordado mediante estas. Este tipo de encuestas suelen ser encuestas por muestreo en las cuales se selecciona una fracción de la población sobre la cual se recolecta la información y luego se extrapolan las conclusiones al total (Schuschny y Soto, 2009).

Por su parte, el objetivo de PISA es evaluar a estudiantes de 15 años (momento en que están próximos a culminar el nivel educativo medio) acerca de si han adquirido las competencias necesarias para desarrollar su vida adulta. Cabe aclarar que este programa considera a alumnos escolarizados en un determinado curso académico, sino que comprende a todos los que tengan entre 15 años y tres meses y 16 años y dos meses de edad al momento de llevarse a cabo el operativo. La muestra se estima por medio de un procedimiento en dos etapas: en la primera se selecciona cierta cantidad de centros educativos (se requieren como mínimo 150 por país), y en la segunda se eligen aleatoriamente 35 alumnos en cada centro. Finalmente, luego se descartar las y los alumnos que por causas acordadas internacionalmente no participan, el número de estudiantes evaluados en cada país debe ser superior a 4500.

PISA se implementa desde el año 2000 cada tres años, la última ronda fue en 2018, pero la información correspondiente no se encuentra disponible al momento de realizar este trabajo. En cada una de estas se evalúan tres competencias: lengua, matemáticas y ciencias (OCDE, 2014). Cabe señalar que se clasifica como una prueba estandarizada de aprendizaje. Una prueba siempre busca evaluar un resultado, lo que distingue a las estandarizadas es que tienen una aplicación amplia, es decir en diversas situaciones, y que se desarrollan a partir de un conjunto de instrucciones y procedimientos que son estándar. Si bien este tipo de pruebas no está libre de críticas (Llach, Montoya y Roldán, 1999), no se ha encontrado aún una fuente estadística alternativa para evaluar la calidad educativa. Por tal motivo, la información provista por esta prueba es aquí utilizada como aproximación de calidad. 
Si bien el programa proporciona datos a nivel de individuos y de países, en este trabajo interesan los correspondientes al nivel países. En cuanto a los resultados educativos, PISA informa la puntuación promedio que cada país alcanza en cada una de las competencias evaluadas y también cómo se distribuye la población en siete niveles, los cuales van desde debajo de nivel 1 hasta nivel 6, siendo este el que representa el mejor de los resultados. Asimismo, un equipo de expertos determina que aquellos que no logran alcanzar el nivel 2 no poseen las competencias necesarias para desarrollar su vida adulta.

\subsection{Metodología}

A partir del conjunto de índices presentados, se escoge trabajar con el IIEB propuesto por Formichella (2014) porque, como ya se ha mencionado, es el único que busca cuantificar la equidad educativa exclusivamente desde la perspectiva de los resultados, representación aquí considerada. El IIEB está formado, por dos indicadores que se vinculan a la cantidad educativa: a) el porcentaje de individuos entre 25 y 65 años que no posee secundaria completo, b) la tasa de extraedad para individuos entre 12 y 24 años; y, por otra parte, por un indicador que representa logros educativos relacionados a la calidad: c) el porcentaje de individuos que no alcanza el nivel mínimo de competencias para desarrollar su vida adulta según datos de la prueba PISA (nivel 2) en el área de lengua.

En este trabajo se presenta el IIEB con las siguientes modificaciones:

a) En la dimensión vinculada a la cantidad educativa no solo se considera la proporción de individuos entre 25 y 65 años ${ }^{[1]}$ sin secundario completo .pssc), sino que dicho dato se complementa con el indicador inversa de la proporción de educación básica (ipeb). Esto con el fin de poder discriminar entre los diferentes grados de avance promedio de la población en cuanto a educación básica: no posee las mismas competencias un individuo que interrumpe el proceso de su educación básica en el último año que aquel que lo hace en el primero o que ni siquiera comienza el ciclo de educación de nivel medio. Este indicador se define así: 1(años de educación básica promedio/total de años de educación básica) y cuanto más grande es su valor mayor es la inequidad. Un caso de equidad extrema sería aquel en el que toda la población tuviera el total de años de educación básica (12), en dicho caso la inversa de la proporción de educación básica sería igual a cero $(1-12 / 12=0)$.

b) Se omite el indicador tasa de extraedad por dos motivos: a) no hay consenso en que este represente a la dimensión de la cantidad educativa, por ejemplo en el Sistema de Tendencias Educativas en América Latina (SITEAL) es considerado un indicador de eficiencia interna y permanencia en sistema; y b) los individuos con gran atraso escolar tienen altas probabilidades de abandonar el proceso educativo, por ello no es del todo adecuado incluir esta tasa para captar a aquellos que terminarán el ciclo escolar a una edad mayor a la prevista.

c) En la dimensión calidad educativa no se considera únicamente el área de lengua, sino que se brinda información más completa porque se promedian los resultados de las tres competencias evaluadas por PISA: lengua, matemáticas y ciencias.

d) Se introduce una corrección al indicador porcentaje de alumnos que no alcanzan el nivel dos de PISA. La corrección consiste en penalizar a aquellos países que poseen menor acceso al sistema educativo o retraso escolar excesivo, con el fin de que la comparación entre los mismos sea más adecuada. Por ejemplo: un país en el que el acceso es muy restringido puede tener un menor porcentaje de estudiantes por debajo de nivel dos de PISA, pero no porque su sistema educativo es más eficiente, sino porque es más exclusivo y solo asisten quienes tienen condiciones de educabilidad más favorables (Krüger, 2013).

Se opta por introducir una corrección de penalización para incorporar lo explicado en el párrafo anterior, pero evitar suponer que todos aquellos que están excluidos de algún modo no hubieran alcanzado el nivel dos, pues no es posible conocer qué habría sucedido si se hubieran encontrado dentro del sistema educativo. 
Si se denomina . al porcentaje de alumnos que no alcanza el nivel mínimo de competencias y $n c$ a dicho porcentaje corregido, la corrección propuesta es la siguiente: $n c=1-(1-n)^{*} c$; donde . es la tasa de cobertura representada por el índice de cobertura 3 que informa PISA. Este se define como la razón entre las y los alumnos de 15 años elegibles y no excluidos y todos los chicos de 15 años del país. Elegible significa que cursan séptimo grado o más, de este modo el atraso excesivo queda penalizado al corregir por cobertura. No excluidos significa que, además de ser elegibles, los administradores de PISA no los han dejado a un lado de la prueba debido a motivos de discapacidades físicas o intelectuales, ni han quedado fuera por cuestiones de logística o fortuitas (OCDE, 2014). Es particularmente relevante la cuestión de la elegibilidad debido a que puede variar ampliamente entre países (Gamboa y Waltenberg, 2015).

Dada la corrección propuesta, en aquellos países en que la tasa de cobertura es alta, es decir que . se acerca a $1, n c$ es cercano a .; mientras que si . se acerca a cero, $n c$ se aleja de .. Cuanto menor sea la tasa de cobertura, menor es la proporción que queda por encima del nivel dos de PISA (1-n) y mayor el porcentaje corregido de individuos que no posee las competencias mínimas $(n c)$.

En la Tabla 1 pueden observarse los datos que se corresponden con los indicadores hasta aquí mencionados y que serán insumo para el análisis empírico propuesto en el trabajo. 
TABLA 1

Datos utilizados

\begin{tabular}{|c|c|c|c|c|c|c|}
\hline País & $\operatorname{pssc}^{[2]}$ & ipeb & $\begin{array}{l}\text { nc } \\
\text { lectura }\end{array}$ & nc mat & ne cs & $\begin{array}{l}\text { Calidad } \\
\text { (promedio) }\end{array}$ \\
\hline Argentina_00 & 0.59 & 0.26 & 0.57 & $S / D$ & S/D & 0.57 \\
\hline Chile_00 & 0.43 & 0.24 & 0.58 & $S / D$ & S/D & 0.58 \\
\hline Mexico_00 & 0.71 & 0.41 & 0.75 & $\mathrm{~S} / \mathrm{D}$ & $\mathrm{S} / \mathrm{D}$ & 0.75 \\
\hline Brasil_00 & 0.72 & 0.49 & 0.70 & $S / D$ & $S / D$ & 0.70 \\
\hline Peru_00 & 0.83 & 0.37 & 0.90 & $S / D$ & $\mathrm{~S} / \mathrm{D}$ & 0.90 \\
\hline Uruguay_03 & 0.68 & 0.28 & 0.62 & 0.67 & 0.60 & 0.63 \\
\hline Mexico_03 & 0.74 & 0.39 & 0.76 & 0.83 & 0.75 & 0.78 \\
\hline Brasil_03 & 0.88 & 0.47 & 0.73 & 0.87 & 0.76 & 0.79 \\
\hline Argentina_06 & 0.51 & 0.22 & 0.67 & 0.72 & 0.65 & 0.68 \\
\hline Chile_06 & 0.49 & 0.21 & 0.50 & 0.65 & 0.53 & 0.56 \\
\hline Uruguay_06 & 0.72 & 0.27 & 0.63 & 0.63 & 0.60 & 0.62 \\
\hline México_06 & 0.72 & 0.37 & 0.71 & 0.77 & 0.73 & 0.74 \\
\hline Brasil_06 & 0.87 & 0.43 & 0.76 & 0.85 & 0.79 & 0.80 \\
\hline Colombia_06 & 0.83 & 0.39 & 0.73 & 0.83 & 0.76 & 0.78 \\
\hline Argentina_09 & 0.47 & 0.20 & 0.67 & 0.75 & 0.67 & 0.70 \\
\hline Chile_09 & 0.44 & 0.19 & 0.41 & 0.58 & 0.42 & 0.47 \\
\hline Uruguay_09 & 0.71 & 0.29 & 0.63 & 0.67 & 0.64 & 0.65 \\
\hline Mexico_09 & 0.72 & 0.35 & 0.63 & 0.70 & 0.68 & 0.67 \\
\hline Brasil_09 & 0.84 & 0.39 & 0.68 & 0.81 & 0.71 & 0.73 \\
\hline Peru_2009 & 0.69 & 0.29 & 0.74 & 0.81 & 0.77 & 0.77 \\
\hline Colombia_09 & 0.80 & 0.38 & 0.69 & 0.83 & 0.73 & 0.75 \\
\hline Panamá_09 & 0.55 & 0.27 & 0.81 & 0.89 & 0.82 & 0.84 \\
\hline Argentin_12 & 0.45 & 0.18 & 0.63 & 0.73 & 0.61 & 0.66 \\
\hline Chile_12 & 0.40 & 0.16 & 0.44 & 0.60 & 0.46 & 0.50 \\
\hline Uruguay_12 & 0.67 & 0.25 & 0.61 & 0.68 & 0.61 & 0.63 \\
\hline Mexico_12 & 0.69 & 0.33 & 0.63 & 0.71 & 0.67 & 0.67 \\
\hline Brasil_12 & 0.82 & 0.36 & 0.65 & 0.77 & 0.68 & 0.70 \\
\hline Peru_12 & 0.68 & 0.27 & 0.71 & 0.82 & 0.77 & 0.77 \\
\hline Colombia_12 & 0.78 & 0.33 & 0.69 & 0.83 & 0.72 & 0.75 \\
\hline Costa Ri_12 & 0.76 & 0.33 & 0.66 & 0.80 & 0.70 & 0.72 \\
\hline Chile_15 & 0.40 & 0.16 & 0.43 & 0.59 & 0.49 & 0.50 \\
\hline Uruguay_15 & 0.67 & 0.25 & 0.56 & 0.66 & 0.57 & 0.60 \\
\hline Mexico_15 & 0.66 & 0.31 & 0.64 & 0.73 & 0.68 & 0.68 \\
\hline Brasil_15 & 0.80 & 0.34 & 0.65 & 0.79 & 0.69 & 0.71 \\
\hline Peru_15 & 0.69 & 0.28 & 0.66 & 0.75 & 0.69 & 0.70 \\
\hline Colombia_15 & 0.74 & 0.33 & 0.57 & 0.75 & 0.62 & 0.65 \\
\hline Costa Ri_15 & 0.73 & 0.32 & 0.62 & 0.85 & 0.67 & 0.71 \\
\hline Rep.Dom_15 & 0.62 & 0.34 & 0.81 & 0.93 & 0.87 & 0.87 \\
\hline
\end{tabular}

Fuente: elaboración propia en base a OCDE (2014) y OCDE (2016) y a CEDLAS (2012).

[2] En el caso de Argentina, dado que su encuesta de hogares (Encuesta Permanente de Hogares) sólo es representativa del sector urbano, se introdujo una corrección: el valor p informado surge del promedio entre el dato brindado por la EPH (ponderado por la proporción de población urbana según el Censo 2010) y el dato del censo para el sector rural (ponderado por la proporción de población rural según el censo 2010).

e) Se estiman ponderadores para cada dimensión (cantidad y calidad) con el fin de aumentar el grado de objetividad del indicador. Así, los ponderadores surgen por medio de un proceso y no como decisión de cada investigador, a diferencia de lo que ocurre con el Índice de Ponderación del Analista (IPA) presentado en 
Formichella (2014). Para estimar los ponderadores se utiliza la metodología de Análisis de Componentes Principales (ACP). El objetivo de este es explicar, a partir de un grupo de variables, la mayor parte de la variabilidad total de las mismas a través del menor número posible de componentes, perdiendo la menor cantidad de información (Uriel, 1985, citado en Schuschny y Soto, 2009). Un componente representa un grupo homogéneo de variables que tienen una gran correlación entre sí y, en principio, es independiente del resto de los componentes (International Business Machines, 2011). Luego, el método asigna pesos relativos a cada componente y con estos puede formarse un índice de manera tal que su representatividad sea máxima, dado que constituye la mayor proporción posible de la varianza grupal de las variables (Alderete, 2012).

En la Tabla 2 se muestra una comparación entre el IIEB-2014 y el IIEB-2019 aquí propuesto:

TABLA 2

Resumen comparativo entre IIEB-2014 e IIEB-2019.

\begin{tabular}{lll}
\hline IIEB & IIEB-2014 & IIEB-2019 \\
\hline Cantidad & $\begin{array}{l}\text { Proporción de individuos } \\
\text { entre 25 y 65 años sin } \\
\text { secundario completo }\end{array}$ & $\begin{array}{l}\text { Proporción de individuos entre 25 y } \\
\text { 65 años sin secundario completo }\end{array}$ \\
\cline { 2 - 3 } & Tasa de extraedad & $\begin{array}{l}\text { Inversa de la proporción de } \\
\text { educación básica }\end{array}$ \\
\hline Calidad & $\begin{array}{l}\text { Resultados PISA en lengua: } \\
\text { porcentaje que no alcanza } \\
\text { nivel 2 }\end{array}$ & $\begin{array}{l}\text { Resultados PISA en lengua, } \\
\text { matemáticas y ciencias: porcentaje } \\
\text { que no alcanza nivel 2. }\end{array}$ \\
\cline { 2 - 3 } & & Corrección por tasa de cobertura \\
\hline Pond. & $\begin{array}{l}\text { Indice de Ponderación del } \\
\text { Analista }\end{array}$ & Análisis de Componentes Principales \\
\hline
\end{tabular}

Fuente: Elaboración propia.

Con respecto al Análisis de Componentes Principales (ACP), cabe mencionar que es necesario disponer de cierto número de casos para poder llevarlo a cabo. Al no haber consenso acerca de dicho número, existen diferentes reglas, una de ellas se denomina tres a uno, lo que significa que el número de casos debe ser el triple que el de variables (Schuschny y Soto, 2009). Dada la información disponible, es la regla utilizada en este trabajo. Así, las variables a analizar son tres: a) porcentaje de individuos entre 25 y 65 años que no poseen secundario completo; b) inversa de la proporción de educación básica y c) promedio del porcentaje de alumnos que no llega al nivel dos de PISA en cada competencia; y se busca que en cada año bajo estudio haya nueve casos. Para ello, en aquellos períodos que no se alcanza a tener nueve casos, se completa con información correspondiente al año más cercano ${ }^{[3]}$. Sin embargo, no es posible hacer esto en los años 2000 y 2003 por la baja cantidad de países latinoamericanos participantes de PISA en dichos años, lo cual hace insuficiente el número de casos, aún si se intenta completarlos con datos de años cercanos.

Cabe aclarar que, si bien lo más habitual es utilizar esta metodología cuando el número de variables es de gran magnitud, en este caso es igualmente pertinente recurrir a ella. Esto se fundamenta en que la principal razón para considerarla no es lograr la reducción de dimensiones (ya que solo se puede pasar de tres variables a dos dimensiones), sino obtener una ponderación para las dos dimensiones definidas, en función del porcentaje de varianza que cada una explica a partir de los datos originales.

En términos resumidos, las componentes principales son combinaciones lineales de las variables analizadas. Estas componentes pueden calcularse por medio de diferentes softwares, aquí se utiliza el IBM SPSS Statistics 19 y se ordenan en función de cuánto de la varianza conjunta explican ${ }^{[4]}$. 


\section{Resultados}

\subsection{Análisis de componentes principales}

Como ya se ha mencionado en la metodología, se desarrolla un Análisis de Componentes Principales (ACP) con el fin de determinar qué ponderación se les asigna a las dimensiones calidad y cantidad del índice de inequidad educativa basado en logros escolares. Este análisis se elabora para cada período separadamente y también se lleva a cabo el mismo ejercicio en forma conjunta, es decir considerando cada combinación de país-año como una observación.

El primer resultado que se analiza en cada una de las muestras estudiadas es la Prueba de Esfericidad de Bartlett, la cual indica si es pertinente utilizar una ACP. Esta prueba propone la hipótesis nula de que la matriz de coeficientes de correlación entre las variables no es significativamente diferente a la matriz identidad, lo cual quiere decir que no hay relación entre las variables y, por ende, no podrían armarse dimensiones a partir de variables correlacionadas. Esto implica que se busca rechazar la hipótesis nula para poder afirmar que es oportuno utilizar un ACP (International Business Machines, 2011). Como puede observarse en la Tabla 3, el pvalue (probabilidad de cometer error de tipo 1, es decir rechazar la hipótesis nula siendo esta verdadera) que acompaña al estadístico propuesto por Bartlett para llevar a cabo el test es inferior a 0,05 en cada uno de los años considerados separadamente y también en el análisis conjunto. Por lo tanto, con un nivel de confianza del 95\% es posible rechazar la hipótesis nula y afirmar que es adecuado efectuar un ACP.

TABLA 3

KMO y prueba de Bartlett.

\begin{tabular}{|l|l|l|l|l|l|l|}
\hline \multicolumn{2}{|l|}{} & '06 & '09 & '12 & ‘15 & ‘00-‘15 \\
\hline $\begin{array}{l}\text { Medida de adecuación muestral de Kaiser-Meyer- } \\
\text { Olkin. }\end{array}$ & 0.50 & 0.56 & 0.57 & 0.65 & 0.58 \\
\hline $\begin{array}{l}\text { Prueba de } \\
\text { esfericidad } \\
\text { de Bartlett }\end{array}$ & Chi-Cuadrado aproximado & 15.8 & 18.4 & 16.1 & 15.0 & 59.5 \\
\cline { 2 - 8 } & glg.(p-value) & 3 & 3 & 3 & 3 & 3 \\
\hline
\end{tabular}

Fuente: Elaboración propia en base a Análisis de Componentes Principales.

Otra prueba que tiene el mismo objetivo es la Medida de Adecuación Muestral propuesta por KaiserMeyer-Olkin (KMO) (López y Gutiérrez, 2019). Esta analiza el grado de correlación entre dos variables quitando el efecto del resto, ya que puede que dos variables se relacionen por influencia de terceras. Se busca que el valor de la medida KMO sea alto, cuanto más cercano a 1 más adecuado es desarrollar un ACP y se desaconseja si esta es menor a 0,5 (International Business Machines, 2011). Como también puede observarse en la Tabla 3, los valores de KMO indican que es pertinente utilizar un ACP en todos los años considerados y en el análisis conjunto, coincidiendo con la conclusión provista por la Prueba de Esfericidad de Bartlett.

El siguiente paso consiste en observar las componentes que se han formado y, con el fin de que la correlación de cada una de las variables se acerque al valor uno solamente en uno de los factores ( $y$ al valor cero en cuanto al resto), se elabora una transformación la cual consiste en rotar los ejes de los componentes, se denomina Varimax y fue desarrollada por Kaiser en 1958 (Carmona, 2014). En este caso de estudio, en cada uno de los análisis se han formado dos componentes, la primera está compuesta por las variables Proporción de individuos sin secundario completo (pssc) e Inversa de la proporción de educación básica (ipeb), ambas relacionadas a la cantidad educativa; mientras que la segunda se conforma por la variable calidad (c) (ver Tabla 4). 
TABLA 4

Matriz de componente rotado ${ }^{a}$

\begin{tabular}{|c|c|c|c|c|c|c|c|c|c|c|}
\hline \multirow{3}{*}{$\begin{array}{l}\text { Muestra } \\
\text { Variables }\end{array}$} & \multicolumn{2}{|l|}{2006} & \multicolumn{2}{|l|}{2009} & \multicolumn{2}{|l|}{2012} & \multicolumn{2}{|l|}{2015} & \multicolumn{2}{|c|}{$2000-2015$} \\
\hline & \multicolumn{2}{|c|}{ Comp. } & \multicolumn{2}{|c|}{ Comp. } & \multicolumn{2}{|c|}{ Comp. } & \multicolumn{2}{|c|}{ Comp. } & \multicolumn{2}{|c|}{ Comp. } \\
\hline & 1 & 2 & 1 & 2 & 1 & 2 & 1 & 2 & 1 & 2 \\
\hline pssc & .972 &, 182 & .973 & .193 & .964 & 218 &, 932 & 316 & 895 &, 357 \\
\hline ipeb & , 901 & , 397 &, 961 & ,246 & ,934 & ,316 & , 822 & , 518 & ,956 &, 174 \\
\hline C & ,262 & 964 &, 220 & 975 & ,265 & 964 & , 374 & ,925 &, 252 & .966 \\
\hline 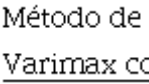 & & & & & & & & & & \\
\hline
\end{tabular}

Fuente: elaboración propia en base a Análisis de Componentes Principales

Fuente: elaboración propia en base a Análisis de Componentes Principales

Así, en la Tabla 5 puede observarse qué proporción de la varianza total de los indicadores que forman parte del Índice, logra explicar cada componente. Es decir, cuánto de la varianza de dichos indicadores puede ser explicado por cada componente. Luego, a partir de este dato puede estimarse el porcentaje de varianza qué cada componente representa en relación al total de varianza explicada acumulado. De allí surge que, en todas las muestras analizadas, el factor correspondiente a la cantidad educativa explica un $60 \%$ y el correspondiente a calidad un $40 \%{ }^{[5]}$. Por tal motivo, al calcular el IIEB se pondera con 0,6 a los resultados vinculados a la cantidad educativa y con un 0,4 a los vinculados a la calidad educativa. Dado que la explicación de la varianza se informa por componente, al interior de cada una se supone una ponderación idéntica para cada variable involucrada.

TABLA 5

Porcentaje de varianza Explicado por cada componente.

\begin{tabular}{|c|c|c|c|c|c|}
\hline \multirow{2}{*}{$\begin{array}{l}\text { Países } \\
\text { Latinoamericanos }\end{array}$} & \multirow{2}{*}{ Comp. } & \multicolumn{3}{|c|}{$\begin{array}{l}\text { Suma de las saturaciones al } \\
\text { cuadrado de la rotación }\end{array}$} & \multirow{2}{*}{$\begin{array}{l}\text { Porcentaje de } \\
\text { varianza explicada } \\
\text { en relación al total } \\
\text { acumulado }\end{array}$} \\
\hline & & Total & $\%$ var. & $\%$ acum. & \\
\hline \multirow{2}{*}{$\begin{array}{l}\text { Participantes en } \\
\text { PISA } 2006\end{array}$} & 1 & 1.826 & 60.874 & 60.874 & 0,62 \\
\hline & 2 & 1.120 & 37.345 & 98.219 & 0.38 \\
\hline \multirow{2}{*}{$\begin{array}{l}\text { Participantes en } \\
\text { PISA } 2009\end{array}$} & 1 & 1.919 & 63.972 & 63.972 & 0.65 \\
\hline & 2 & 1.049 & 34.980 & 98.952 & 0.35 \\
\hline \multirow{2}{*}{$\begin{array}{l}\text { Participantes en } \\
\text { PISA } 2012\end{array}$} & 1 & 1.872 & 62.394 & 62.394 & 0.63 \\
\hline & 2 & 1.077 & 35.905 & 98.299 & 0.37 \\
\hline \multirow{2}{*}{$\begin{array}{l}\text { Participantes en } \\
\text { PISA } 2015\end{array}$} & 1 & 1.684 & 56.120 & 56.120 & 0.58 \\
\hline & 2 & 1.223 & 40.761 & 96.881 & 0.42 \\
\hline \multirow{2}{*}{$\begin{array}{l}\text { Participantes en } \\
\text { PISA 2003-2015 }\end{array}$} & 1 & 1.778 & 59.271 & 59.271 & 0.62 \\
\hline & 2 & 1.091 & 36.369 & 95.640 & 0.38 \\
\hline
\end{tabular}

Fuente: elaboración propia en base a Análisis de Componentes Principales

\subsection{Análisis del IIEB-2019}

A partir de los resultados del IIEB obtenidos -los cuales pueden observarse en la Tabla 6 y en el Gráfico 1se evidencia que, dentro de la muestra analizada, el país con menor nivel de inequidad educativa es Chile en todos los años considerados, lo cual coincide con lo hallado por Formichella (2014). Mientras que Argentina y Uruguay son los países que más de cerca lo siguen en casi todos los períodos. Por otra parte, se verifica 
que México, Brasil, Perú, República Dominicana, Panamá, Costa Rica y Colombia presentan, en términos relativos, mayores dificultades en cuanto a la equidad educativa.

TABLA 6

Valor de IIEB-2019 por país y por año.

\begin{tabular}{lccccccc}
\hline País/IIEB & 2000 & 2003 & $\mathbf{2 0 0 6}$ & $\mathbf{2 0 0 9}$ & $\mathbf{2 0 1 2}$ & $\mathbf{2 0 1 5}$ & Variación $^{\text {[6] }}$ \\
\hline Argentina & 0.48 & & 0.48 & 0.44 & 0.43 & & -11.13 \\
\hline Brasil & 0.64 & 0.72 & 0.68 & 0.63 & 0.62 & 0.58 & -9.74 \\
\hline Chile & 0.43 & & 0.42 & 0.36 & 0.34 & 0.20 & -53.40 \\
\hline Colombia & & & 0.67 & 0.64 & 0.62 & 0.55 & -18.12 \\
\hline Costa Rica & & & & & 0.55 & 0.61 & 10.90 \\
\hline México & 0.63 & 0.65 & 0.62 & 0.58 & 0.56 & 0.55 & -13.38 \\
\hline Panamá & & & & 0.55 & & & \\
\hline Perú & 0.72 & & & 0.59 & 0.57 & 0.62 & -13.64 \\
\hline Uruguay & & 0.54 & 0.52 & 0.52 & 0.50 & 0.41 & -24.34 \\
\hline $\begin{array}{l}\text { República } \\
\text { Dominicana }\end{array}$ & & & & & & 0.66 & \\
\hline
\end{tabular}

Fuente: elaboración propia

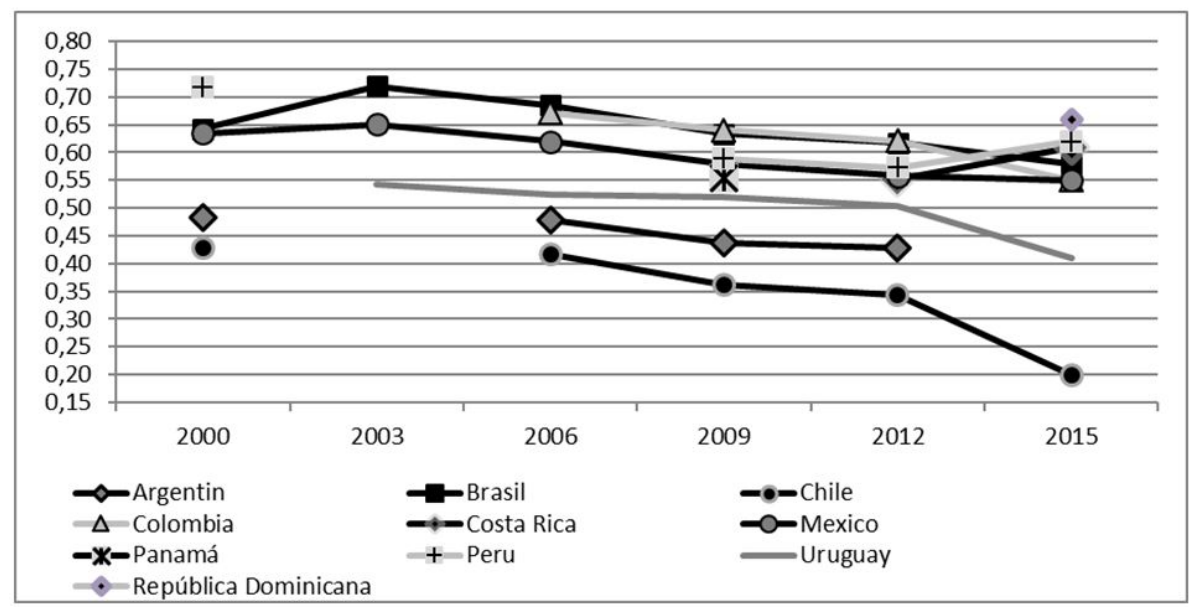

GRÁFICO 1

IIEB-2019 por país 2000-2015.

Fuente: Elaboración propia

Un dato interesante que surge de la observación del Gráfico 1 es que el ranking entre países prácticamente no se ha modificado. Como se verifica en la Tabla 6, todos los países, excepto Costa Rica, han mejorado sus niveles de equidad educativa. Además, los países que lo han hecho con mayor intensidad son aquellos que se encontraban en la cima de la equidad. Esto significa que las mejoras al interior de cada país han sucedido a un ritmo que no ha implicado un cambio sustancial en las posiciones relativas entre estos. El único país que pareciera ha subido en el ranking de la equidad es Uruguay, pues en 2015 obtuvo un valor del IIEB menor a Argentina en 2012. Lamentablemente, al no contarse con datos para Argentina $2015^{[7]}$ no puede tenerse certeza de este cambio relativo.

De todas maneras, se destaca que Argentina, junto a Brasil, ha sido de los países que menor éxito ha tenido en la disminución del valor del IIEB. Mientras que Chile y Uruguay, con quienes compartía la cima de la equidad educativa, han tenido una performance claramente mejor (Chile bajo su nivel de inequidad en un $53.4 \%$ entre 2000 y 2015 , mientras que Uruguay lo hizo en un $24.34 \%$ ). 
A continuación, se presenta la Tabla 7 con el ordenamiento relativo de los países en cada período de forma ascendente en términos de equidad.

TABLA 7

Posiciones relativas de cada país en cada uno de los años analizados.

\begin{tabular}{lllllll}
\hline $\begin{array}{l}\text { Equidad } \\
\text { Educ. } \\
\text { Puesto }\end{array}$ & 2000 & 2003 & 2006 & 2009 & 2012 & 2015 \\
\hline $\mathbf{1}$ & Chile & Uruguay & Chile & Chile & Chile & Chile \\
\hline $\mathbf{2}$ & Arg. & México & Arg. & Arg. & Arg. & Uruguay \\
\hline $\mathbf{3}$ & México & Brasil & Uruguay & Uruguay & Uruguay & México \\
\hline $\mathbf{4}$ & Brasil & & México & Panamá & Costa R. & Brasil \\
\hline $\mathbf{5}$ & Perú & & Brasil & México & México & Colombia \\
\hline 6 & & & Col. & Perú & Perú & Perú \\
\hline 7 & & & & Brasil & Col-Brasil & Rep. Dom. \\
\hline 8 & & & & Col. & & \\
\hline
\end{tabular}

Fuente: Elaboración propia

En la Tabla 7 también se observa que existe un grupo de países que ha tenido una mayor continuidad en la participación en el Programa PISA. En particular, se consideran aquellos que han estado presentes en al menos cuatro rondas. De esta manera, es posible disponer de su valor de IIEB, como mínimo, para cuatro de los períodos considerados. Este subgrupo de países puede dividirse en dos: a) aquellos que mayor nivel de equidad educativa han obtenido en los años estudiados (ver Gráfico 2), y b) los que menor nivel de equidad han verificado en dichos períodos (ver Gráfico 3).

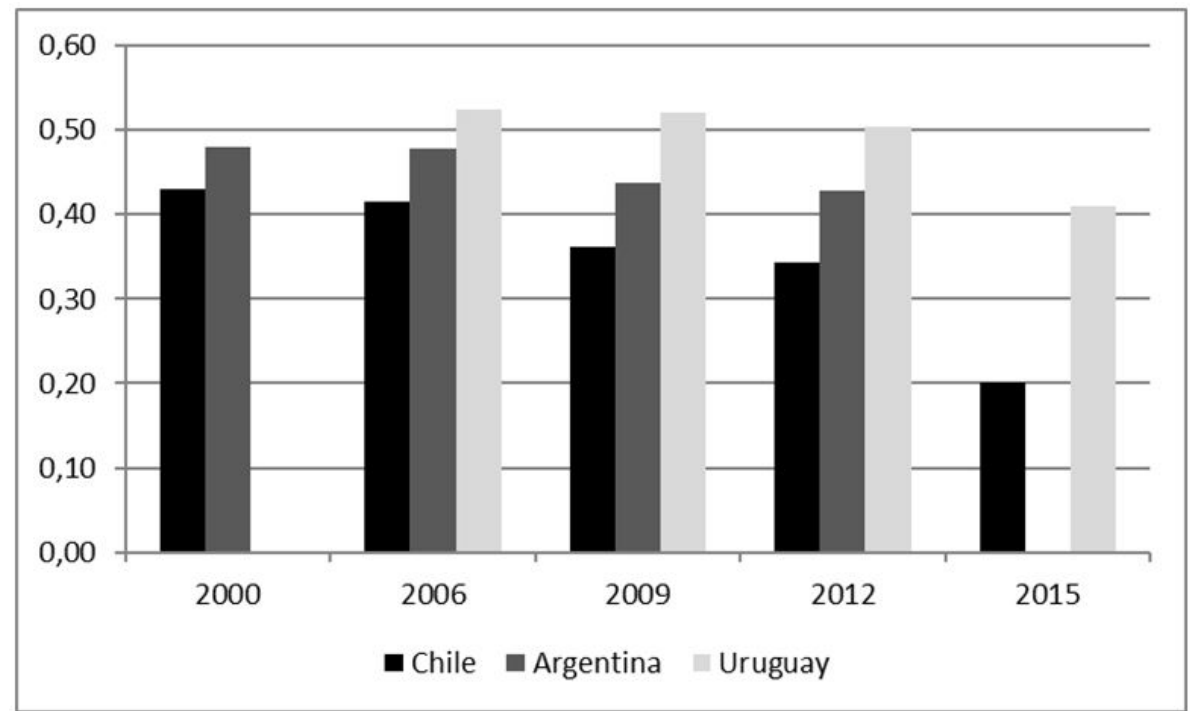

GRÁFICO 2

IIEB para los países con mayor equidad educativa Fuente: Elaboración propia

En el gráfico anterior se observa que, del grupo de países más equitativos en educación, en los últimos dos períodos considerados todos lograron situarse por debajo del nivel medio de inequidad $(0,5)$ y que las posiciones relativas entre ellos no han cambiado en los años estudiados. 


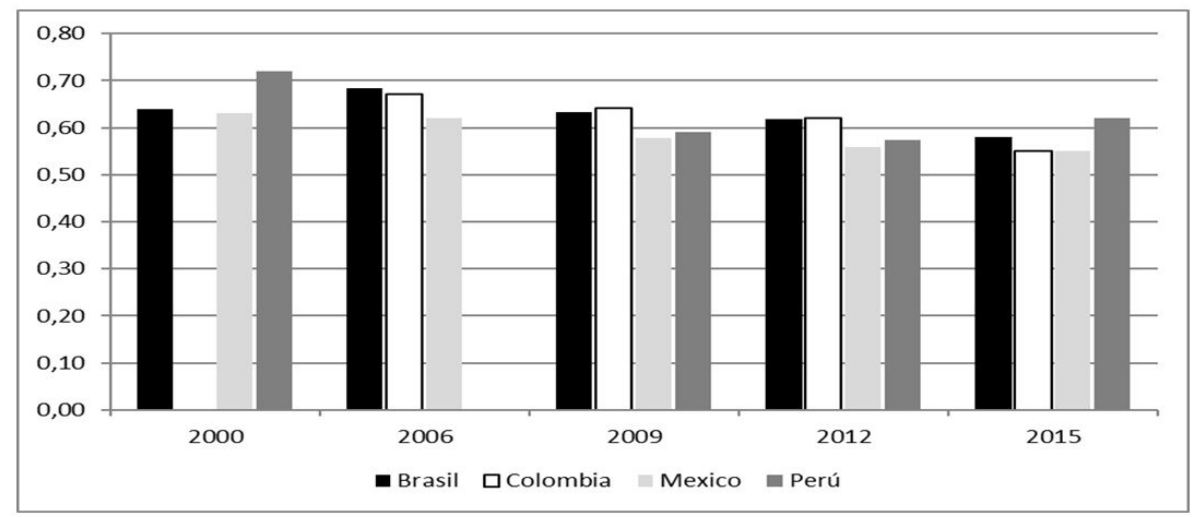

GRÁFICO 3

IIEB para los países con menor equidad.

Fuente: Elaboración propia

El Gráfico 3 muestra que los países menos equitativos superan en todos los períodos el nivel medio de inequidad y que las posiciones relativas entre ellos, al igual que en subconjunto mencionado anteriormente, prácticamente no se han modificado en los años analizados.

Asimismo, si se consideran al mismo tiempo los resultados de estos dos conjuntos de países puede obtenerse un valor promedio del IIEB de los más equitativos y compararse con el promedio del IIEB de los menos equitativos. De este modo, puede calcularse cuál es la brecha entre ambos grupos para cada período estudiado. Esto puede observarse en el Gráfico 4:

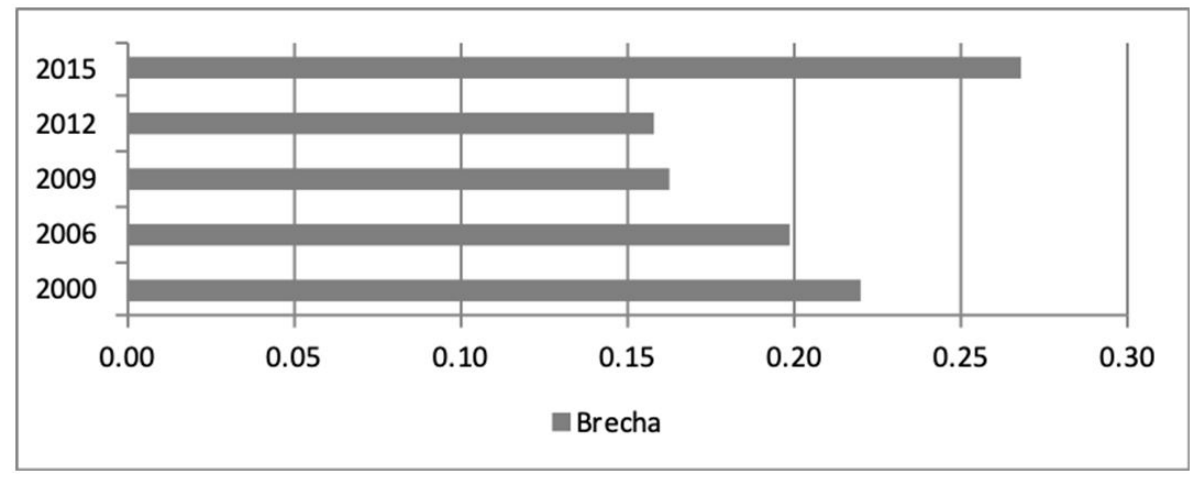

GRÁFICO 4

Brecha entre el grupo de países más y menos equitativos.

Fuente: Elaboración propia

Queda en evidencia en el Gráfico 4 que, si bien la distancia entre los dos conjuntos de países fue disminuyendo durante los primeros años estimados, mostró un importante aumento en el año 2015. Se destacan dos cuestiones relevantes: por un lado, la brecha creció un 22\% entre el año 2000 y el 2015 y, por el otro, el último dato disponible muestra que la inequidad promedio en el grupo de los menos igualitarios (Valor Promedio del IIEB $=0,57$ ) es un $88 \%$ más elevada que en el grupo de los más igualitarios (Valor Promedio del IIEB=0,31).

Si el mismo análisis se lleva a cabo en términos individuales, calculando la brecha entre el país menos equitativo y el más equitativo de esta submuestra, también se observa una ampliación de la distancia en el último período considerado, 2015 (ver Gráfico 5). 


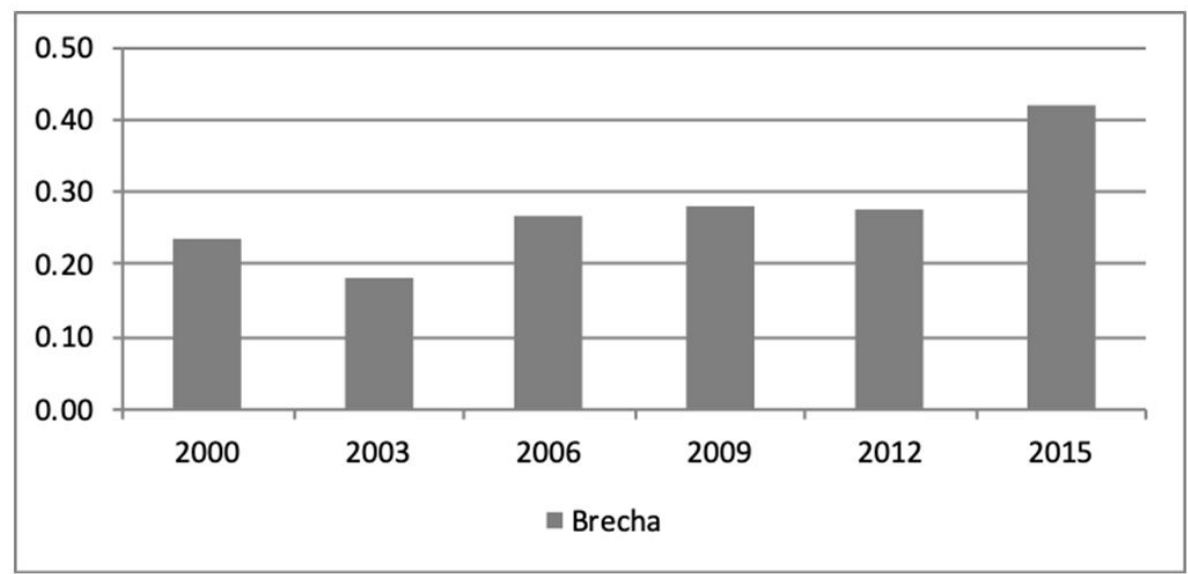

GRÁFICO 5

Brechas entre países en cada año.

Fuente: Elaboración propia

Lo que se observa en el Gráfico 5, se corresponde con lo mencionado párrafos atrás acerca de que Chile y Uruguay, países pertenecientes al grupo de los más igualitarios, han sido los que proporcionalmente han mejorado la inequidad educativa en mayor grado.

De este modo, puede decirse que no hay evidencia de que al interior de la submuestra de países latinoamericanos aquí analizados -es decir aquellos que han presentado continuidad en su participación en PISA- se observe una tendencia hacia una convergencia en términos de equidad educativa, sino al contrario.

\section{Consideraciones FinALES}

A lo largo de este trabajo se ha intentado describir la situación de América Latina en cuanto a la equidad educativa en los años 2000, 2003, 2006, 2009, 2012 y 2015, en función de los datos disponibles. Para ello, se utilizó el IIEB-2014 presentado por Formichella (2014) y se le introdujeron modificaciones con el fin de cumplir con el objetivo propuesto, dando lugar al IIEB-2019.

El nuevo índice propuesto aparece como una versión superadora del anterior debido a las siguientes cuestiones: en relación a la cantidad educativa de la población, no se limita en discriminar entre quienes han culminado la educación básica y quienes no, sino que permite distinguir entre los diferentes grados de avance promedio; penaliza a los países cuyos sistemas educativos son menos inclusivos, al incorporar el indicador de cobertura; es más amplio porque incluye las tres competencias de PISA al momento de estimar la calidad; y es más objetivo porque utiliza ponderadores que surgen a partir de una metodología específica.

Como consideraciones finales se resaltan cuatro cuestiones. En primer lugar, se observa que todos los países estudiados, excepto Costa Rica, han verificado una disminución en su grado de inequidad educativa. Sin embargo, se destaca el bajo nivel de éxito de Brasil y Argentina en relación a este objetivo.

En segundo lugar, es importante notar que este resultado positivo no debe ocultar el hecho de que los últimos valores del IIEB-2019 disponibles, correspondientes al año 2015, son extremadamente altos en la mayor parte de los países, superando el valor medio $(0,5)$ en prácticamente todos los casos, quedando fuera solo Chile y Uruguay. Esto significa que el diagnóstico es preocupante y que debe ser un punto prioritario en las agendas de política de los países latinoamericanos.

En tercera instancia, cabe mencionar que, si bien todos los países han verificado una mejora en términos de equidad, los que partieron de una posición más favorable coinciden con aquellos que fueron más exitosos, logrando disminuir la inequidad en mayor proporción (se destacan los casos de Chile y Uruguay). Esto 
implica que el ranking entre países prácticamente no se ha modificado y que la brecha entre los más y los menos equitativos ha aumentado en el período analizado.

Por último, se observa que la variabilidad entre los países está marcada por la cantidad educativa (60\%) en mayor proporción que por la calidad (40\%). Sin embargo, esto no significa que los hacedores de política deban poner atención a la cuestión de la cantidad en detrimento de la calidad, ya que la poca variabilidad en calidad se verifica en valores muy bajos de la misma. En 2015, el promedio de la proporción de estudiantes que no alcanza los niveles mínimos de habilidades para desarrollar su vida adulta - considerando las tres competencias evaluadas por PISA y corrigiendo su valor por tasa de cobertura- supera el valor medio en todos los países estudiados, incluso en Chile que lleva la delantera. Es decir que, como puede observarse en la Tabla 1, nc es mayor a 0,5 en todos los períodos y en todos los casos analizados, lo cual representa un diagnóstico sumamente preocupante a ser tenido en cuenta al momento de tomar decisiones políticas.

En síntesis, a partir de este trabajo se ha cuantificado la inequidad educativa en América Latina en los años señalados, se ha establecido un ranking de países y se ha analizado la brecha entre los mismos. De esta manera, se espera haber contribuido a darle mayor visibilidad a la problemática y se recomienda continuar con la recolección de información y con el cálculo de la inequidad educativa, por ser sumamente útil al momento de hacer un diagnóstico de la situación.

Por último, cabe señalar que en investigaciones futuras se ampliará la muestra de países con el fin de analizar la posición de América Latina en relación al resto del mundo.

\section{Referencias BibliográficAs}

Alderete, M. (2012). Medición de las Tecnologías de la Información y la Comunicación en empresas de servicios de Colombia. Cuadernos de Administración, 25(45), 39-62.

Assiego, V.y Ubrich, T. (2015). Iluminando el futuro. Invertir en educación es luchar contra la pobreza infantil. Madrid, España: ave the Children.

Bolívar, A. (2005). Equidad educativa y teorías de la justicia. Revista electrónica iberoamericana sobre calidad, eficacia y cambio en educación, .(2), 42-69.

Calero J. (1999) Indicadores de equidad interna y externa en la educación superior. Metodologías y una aplicación al caso español. En M. Kisilevsky (Ed.) Indicadores universitarios: tendencias y experiencias internacionales (pp.151-171). Buenos Aires, Argentina: EUDEBA.

Carmona, F. (13 de enero de 2014). Un ejemplo de APC paso a paso. [Documento de trabajo]. Recuperado de http:/ /www.ub.edu/stat/docencia/Mates/ejemploACP.PDF

Carvalho, M., Gamboa, L. y Waltenberg, F. (2015). Equality of educational opportunity: Taking both achievement and access into account .Ecineq working paper 2012-277). IBGE. Recuperado de http://www.iariw.org/papers/2013 /CarvalhoPaper.pdf

Castillo, C. (2003) La equidad en el sistema educativo en Honduras. Colección Cuadernos de Desarrollo Humano Sostenible 17. Honduras: Programa de las Naciones Unidas para el Desarrollo. Tegucigalpa, Honduras: PNUD.

Castro, D. (2019) Equidad en la Distribución de Recursospara la Educación Colombiana. [Tesis de Maestría en Ciencias Económicas]. Universidad Santo Tomás, Bogotá, Colombia.

Centro de Estudios Distributivos, Laborales y Sociales [CEDLAS] (2012). Guia SEDLAC. Base de datos socioeconómicos para América Latina y el Caribe. Recuperado de http://www.cedlas.econo.unlp.edu.ar/wp/esta disticas

Chávez H, E., E., Abreu L., R. y Charles, A. (2013). Índice de Equidad Educativa Indigena Informe de Resultados para México, sus Estados y Municipios, 2010. Ciudad de México, México: PNUD.

Cohen, G. A. (1989). On the currency of egalitarian justice. Ethics, 99(4), 906-944.

Dworkin, R. (1981). What is equality? Part 2: equality of resources. Philosophy and public affairs, 10(3), 283-345.

Fleurbaey, M. (1995). Equality and responsibility. European Economic Review, 39, 683-689. 
Formichella, M. (2010) Educación y desarrollo: Análisis desde la perspectiva de la equidad educativa interna y del mercado laboral (Tesis de doctorado). Universidad Nacional del Sur, Bahía Blanca, Argentina.

Formichella, M. (2011) Análisis del concepto de equidad educativa a la luz del enfoque de las capacidades de Amartya Sen. Educación, 35(1), 1-36. https://doi.org/10.15517/REVEDU.V35I1.25150

Formichella, M. (2014) Equidad educativa: Medición y aplicación a Latinoamérica. Education Policy Analysis Archives, 22(1), 1-26

Gamboa, L. y Waltemberg, F. (2012) Inequality of opportunity for educational achievement in Latin America: Evidence from PISA 2006-2009. Economics of Education Review, 31, 694-708.

Gamboa, L. y Waltemberg, F. (2015). Measuring Inequality of Opportunity in Education by Combining Information on Coverage and Achievement in PISA, Educational Assessment, 20(4), 320-337.

International Business Machines (2011) Manual del usuario del sistema básico de IBM SPSS Statistics 20. Nueva York, Estados Unidos: IBM

Krüger, N. (2013) Equidad educativa interna y externa en Argentina: un análisis para las últimas décadas (Tesis de doctorado). Universidad Nacional del Sur, Bahía Blanca, Argentina.

Llach, J., Montoya, S. y Roldán, F. (1999). Educación para todos. Buenos Aires, Argentina: IERAL.

López, N. (2006). Equidad educativa y desigualdad social. Buenos Aires, Argentina: IIPE-UNESCO.

López A, M. y Gutiérrez P, L. (2019). Cómo realizar e interpretar un análisis factorial exploratorio utilizando SPSS. REIRE Revista d'Innovació i Recerca en Educació, 12(2), 1-14.

Mondéjar J, J y Vargas V, M. (2008). Indicadores sintéticos: una revisión de los métodos de agregación. Revista Economia, Sociedad y Territorio, 3(27), 565-585.

Montes, N. (2004). Adolescentes y jóvenes en contexto. El marco cercano: la familia, y el marco amplio: los otros. En Guillermina Tiramonti (Ed.) La trama de la desigualdad educativa (pp. 47-73). Buenos Aires, Argentina: Manantial.

Morduchowicz, A. (2003). Discusiones de economía de la educación. Buenos Aires, Argentina: Losada.

Organización para la Cooperación y el Desarrollo Económicos [OCDE]. (2014). PISA 2012. Technical Report. Paris, Francia: OECD Publishing.

Organización para la Cooperación y el Desarrollo Económicos [OCDE]. (2016). PISA 2015 results. Paris, Francia: OECD Publishing.

Pérez G.; Blancas Peral, F.; Gonzáles Lozano, M.; Guerrero Casas, F.; Lozano Oyola, M. y Ruiz Camacho, M (2008) Análisis, diseño y comparación de indicadores sintéticos. En J. Bernal García (Presidencia). XVI Jornadas Asociación Española de Profesores Universitarios de Matemática para la Economia y la Empresa-ASEPUMA-IV Encuentro Internacional. Facultad de Ciencias de la Empresa, Universidad Politécnica de Cartagena (UPCT), Cartagena, Colombia.

Rawls, J. (1971). Teoría de la justicia. Ciudad de México, México: Fondo de Cultura Económica.

Rawls, J. (1999). Justicia como equidad. Revista española de control externo, 5(13), 129-158.

Ribotta, S. (2006) Las desigualdades económicas. Un estudio desde el igualitarismo contemporáneo (Tesis de Doctorado). Universidad Carlos III, Madrid, España.

Roemer, J.(1995). Equality and responsibility. Boston Review Forum, Social Equality and Personal Responsibility, 20(2). Recuperado de http://bostonreview.net/archives/BR20.2/roemer.html

Schuschny, A. y Soto, H (2009). Guía Metodológica. Diseño de Indicadores Compuestos de Desarrollo Sostenible. Santiago de Chile, Chile: Naciones Unidas.

Sen, A. (1979). Equality of What? En O. C. Tanner (presidencia). Tanner Lecture on Human Values. Stanford University. Stanford, California, EE.UU. Recuperado de http://www.ophi.org.uk/wp-content/uploads/Sen-1 979_Equality-of-What.pdf

Sen, A. (1999). Desarrollo y Libertad. Buenos Aires, Argentina: Planeta.

Sen, A. (28 de octubre de 2003). The importante of Basic education. [Conferencia en Edinburgh University]. Recuperado de http://birbhum.gov.in/DPSC/reference/4.pdf 
Shavelson, R., McDonnell, L. y Oakes J. (1991). What Are Educational Indicators and Indicator Systems? En Practical Assessment, Research \& Evaluation, 2(11). Recuperado de https://scholarworks.umass.edu/cgi/viewcontent.cg i? article $=1033 \&$ context $=$ pare

Torres, M. (2010) América Latina: Seis décadas de metas para la educación. [Mensaje en un blog]. Recuperado de htt ps://otra-educacion.blogspot.com/2010/12/america-latina-cuatro-decadas-de-metas_20.html

UNESCO (1966). Proyecto principal para la extensión y mejoramiento de la educación primaria en América Latina. Santiago de Chile, Chile: UNESCO.

\section{Notas}

[1] Se toma este margen de edad porque es el dato disponible de manera homogénea para los países a estudiar.

[2] En el caso de Argentina, dado que su encuesta de hogares (Encuesta Permanente de Hogares) sólo es representativa del sector urbano, se introdujo una corrección: el valor p informado surge del promedio entre el dato brindado por la EPH (ponderado por la proporción de población urbana según el Censo 2010) y el dato del censo para el sector rural (ponderado por la proporción de población rural según el censo 2010).

[3] Así, en 2006 se completa con información de Perú y Panamá 2009 y Costa Rica 2012; en 2009 se completa con Costa Rica 2012; en 2012 con Panamá 2009 y en 2015 con Argentina 2012.

[4] Para mayores detalles ver: Schuschny A. y Soto H. (2009) Guía metodológica. Diseño de indicadores compuestos de desarrollo sostenible. Documento de trabajo de la CEPAL.

[5] Con redondeo de decimales.

[6] Se calcula con los valores extremos disponibles.

[7] Si bien se aplicó el Programa PISA en Argentina en el año 2015, el equipo de la OCDE informa que no pueden utilizarse los resultados por problemas con la muestra de escuelas utilizada (OCDE, 2016).

\section{BY-NC-ND}

